\title{
Multi-dimensional Representations of Laparoscopic Simulations for SANETs
}

\author{
Christopher Chiu and Zenon Chaczko \\ Faculty of Engineering \& IT, University of Technology, Sydney, Australia \\ \{christopher. chiu, zenon. chaczko\}@uts.edu. au
}

\begin{abstract}
This paper describes the development of a Sensor-Actuator Network (SANET) middleware environment to model laparoscopic procedures in a simulated environment. This case study examines the use of software agents to represent the organic subject (where the surgical procedure is performed) and the surgical tool/effector being manipulated by the end user (the surgeon performing the operation). Developed using the Belief-Desire-Intention (BDI) agent-based software reasoning approach by Rao and Georgeff (1995), the interaction of the agent elements in the simulation, coupled with the feedback mechanisms as the operator interacts with the simulated tissue, will aid in the simulation of laparoscopic procedures for medical training.
\end{abstract}

Keywords: Biomimetic Engineering, Medical Simulation, Multidimensional Modelling, Software Agents, Sensor Actuator Networks (SANET).

\section{Introduction}

Embedded in-situ sensors in medical instrumentation is a complimentary technology that provide real-time feedback to health professionals in the examination and diagnosis of patients. Coupled with the miniaturization and inexpensive nature of modern wireless sensors, they will become pervasive in medical apparatus. The emerging technological development for implementing wireless sensor networks in medical equipment provide the following development enhancements [1, 11]:

- Physical Portability: With wires and cables eliminated from the sensory device and the data collection interface, it allows for flexible usage parameters from a design and development point of view. Coupled with a rechargeable base-station and powered by lithium-ion polymer batteries, this will allow the end user of the device to operate it in a seamless manner;

- Communication-Medium Enabler without Physical Bounds: Wireless radio-frequency communication is unconstrained by physical wiring as well as line of sight constraints (i.e. Infrared or optic communication), so medical diagnoses can conveniently be achieved in a remote fashion;

- Device Interactivity and Interconnectivity: Devices sharing a common protocol standard for low-power RF communication (i.e. Bluetooth IEEE 802.15.1, ZigBee IEEE 802.15.4) [2, 7] can inter-communicate with one another, allowing for the aggregation and analysis of medical readings amongst different analytical devices for predictive end-user forecasts; and 
- Smart Heuristics Monitoring and Integration: Health data that is aggregated and collated from different sources can be integrated together using data-fusion heuristics [6, 7], thus allowing professionals to be presented with a medical diagnosis containing the complete picture of health for a patient.

The main aspect to be investigated is the use of an agent-based software framework to utilize the data captured from a wireless laparoscopic probe. This data can represent different forms and means, and be presented to the end user in such a way to provide feedback of an laparoscopic trainee's performance. The main sources of feedback that can be provided to the end user's somatosensory system are as follows [3]:

- Haptic: The operation of the probe will be integrated with force-feedback controllers, where tension or potential detection of strain or stress will result in discrete vibrations or physical resistance against a particular direction;

- Aural: Aural cues to signify that a hazardous zone breach could occur, as the probe could potentially contact with a sensitive area of the body such as vital organs. These cues can vary in tone, frequency of the cue, and the volume to alert the severity of the current situation to the surgeon;

- Visual: Modern probes contain an optic-fiber illuminated CMOS video camera integrated at the probes receptacle, so a first-person view of the surgical procedure takes place [2. It is critical that visual feedback is maintained in a hard real-time manner to ensure all information is presented to the end-user in an instantaneously.

The purpose of this paper is the case study into a visualization mechanism that presents the various data sources as a multi-dimensional construct to the laparoscopic surgeon in training [2, 12]. As the trainee performs a pre-defined procedure, real-time visualization indicators are presented to the user to provide guidance to their current actions and possible rectifications to prevent harm to the patient. The analytical data generated from the training session can be is replayed to the trainee and trainer, so a thorough feedback of the training session is evaluated on merit indicators of precision and expertise.

\section{Visualization as an Agent-Based Process}

Utilizing the Belief-Desire-Intention (BDI) software agent paradigm by Rao and Blackwell [8, a simulation model has been developed for a sensor networkenabled visualization component to model laparoscopic surgical training [3]. The agent-based architecture of the sensor-network middleware model interfaces the physical training hardware (including sensors and actuators) and associates a unique software agent for each apparatus. Each agent will be identified with unique traits, or rules, that govern the normal usage parameters and mode of operation within tolerable limits (as specified by manufacturer data sheets and physical characteristics and properties for each device).

Visualization is the agent process that will be the main focus of the case study. As the operator performs the pre-defined procedure, event-tracking heuristic selection takes place via self-organizing maps (neural networks) [5, 6], such that 
in multi-dimensional space the visualization of the heuristic service in execution can show the SANET network in operation in terms of allometry, ontology and hierarchy 9. The visualization system utilizes OpenGL Hardware-accelerated graphics libraries, to reduce the processing load from the main processor and ensure overall responsiveness of the middleware environment.

In addition to the standard 3D representation of the SANET environment, surface relief maps extend the visualization in a multi-dimensional perspective, with the $\mathrm{X}$ and $\mathrm{Y}$ coordinates relative to the $\mathrm{n}^{\text {th }}$ dimension. This augments the reality of the $3 \mathrm{D}$ space, while enabling the end-user to view the variable dimension being analyzed in the relief map (i.e. energy surface maps, sensory readings or actuator stress points). In summary, the environmental parameters driving the visualization software component combine both viewpoints to incorporate both Euclidean metrics and non-Euclidean space:

\section{- Agent Representations}

Graphically rendered constructs correlate to each software agent in 3D space. By system default, this is defined as the physical position of the agent in Euclidean space; although this can be represented in non-euclidean space as a user specified instruction. The predefined settings for agent representation include 3D Euclidean space, energy plot representations of the mobile actuators and wireless sensors, and the bidirectional signal strength of the wireless sensor communicating to the actuators about the environmental space.

\section{- Surface/Relief Maps}

Surface maps chart a specific attribute to each agent according to an $\mathrm{X}-\mathrm{Y}$ plane; while the $\mathrm{Z}$ plane corresponds to the attribute value. Surface maps incorporate standardized Voronoi tessellation techniques to render the surface map overlays within each agent representation. Surface maps aid in visually identifying regions of interest where risks are likely to occur - including severity of energy depletion and wireless signal 'black spots' - such that preemptive action by a surgical student or intervention by the instructor can be achieved at the earliest opportunity.

\section{Software Agent Suitability to Laparoscopic Surgical Training}

Software applications designed for systemic agent approaches need to factor the following concerns: Feasibility of applying a toolkit in a given domain, such as utilizing a compliant BDI agent system versus hybrid-based models; and consideration of open-source versus closed-source frameworks. For this case study, compatibility with open source platforms was essential to ensure a standardized experimental base, hence the use of Java Standard Edition for the software implementation. The suitability of adapting software agent infrastructures involves framing the domain space within the BDI agent model, and encapsulating the available service agencies in which additional components can be developed:

\section{- Contextual Definition}

As shown by the BDI agency conceptual diagram on the following page (Left), the Plan Library and Belief Set consists of rules in which the bounded 
rational software agent must comply with the physical constraints of the environment space and effector specifications (such as the laws of physics); the Desires define the safety rules are adhered while ensuring a particular target is reached while avoiding obstacles and potential risks; the Intentions are the actualization of the training procedures taking place, inclusive of the haptic human user interface between the system infrastructure and end user.

\section{- Heuristic Integration/Augmentation}

Depicted in the SANET network environment within a Laparoscopic surgical domain on the current page (Right), a software agent environment requires open Application Programming Interface (API) facilities to allow for heuristic integration with hardware devices. A laparoscopic effector can incorporate a variety of pressure and imaging sensors to enable real-time feedback of the operation, from the perspective of the effector itself. Hence, the services incorporated in the current version of the SANET agent infrastructure environment include data persistence services to enable data logging, multimedia records and historical data analysis for post-user feedback, while heuristic service agencies integrate Genetic Algorithm (GA) and Neural Network analytical libraries for image recognition analysis and trajectory tracking problems respectively.



Fig. 1. (Left) Belief-Desire-Intention Agent; (Right) A Laparoscopic SANET-Effector

\subsection{Agent Framework Evaluation}

The software agent systems evaluated for this case study include the XJ AnyLogic Simulation Framework and the Jadex BDI Agent System. Both Java-based frameworks provide the basic libraries and software interfaces to enable development of behavioral subroutines for the laparoscopic surgical domain:

\section{- AnyLogic Simulation Framework by XJ Technologies}

AnyLogic is a closed-source multi-agent paradigm system with neural network development libraries to build agent-based models for scientific and engineering concerns. The system is built with the Eclipse IDE framework for GUI design and ruleset creation, and performs automated code generation for post-project configuration and data analysis. AnyLogic is designed for multi-threading and has fixed 3D models for graphical representation. 

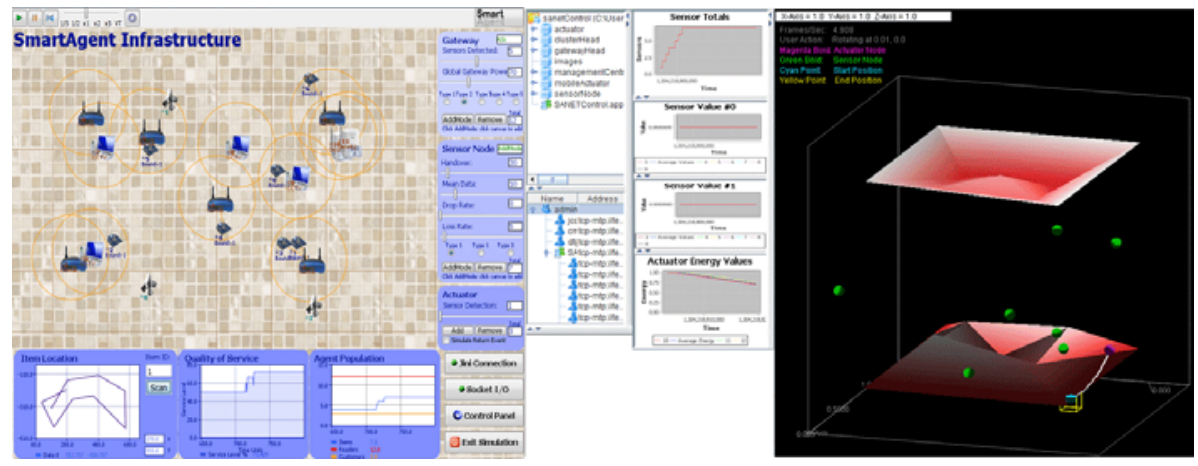

Fig. 2. (Left) AnyLogic Framework; (Right) Jadex Agency with 3D Graphics Services

The screen capture on this page (Left) shows the AnyLogic simulation from a $2 \mathrm{D}$ perspective.

\section{- Jadex BDI Agent System by Universität Hamburg}

Jadex is an open-source Belief-Desire-Intention-compliant reasoning engine based on Jade by Telecom Italia. The underlying framework consists of four components: Jadex Agents as a software framework, Jadex Processes to execute workflow plans, Jadex Rules to define the rule engine and its execution activity, with Jadex XML for data binding and representation. The screen capture on the current page (Right) is the Jadex execution process with a middleware interface for 3D modelling of the SANET environment.

\subsection{Extended Kohonen Map Heuristics Augmentation}

The following algorithmic processes are considered for obstacle detection in a SANET environment for laparoscopic surgical training in the following problem statement tasks [4, 6]:

- Feature Mapping

Process: For initial state described by input vector $u(0)$ in input space $U$ By using a Extended Kohonen Map (EKM) [5, 10], the map self-organizes to partition continuous sensory space into discrete regions. The feature map generalization capability arises from its self-organization during training [5], when the SANET actuator is trained to map a localized sensor region. This approach increases the sensory representation's resolution in the frequently encountered stimuli regions [10].

\section{- Multivariate Regression}

Process: Adapt sequence of control vectors $c(t), t=0, \ldots, T-1$ in sensory control space $C$ with resultant goal state elaborated by $u(T) \in U$

In addition, as a non-linear multi-variate regression problem uninterrupted mapping from $U$ to $C$ is done by training a multilayer perceptron (MLP), which offers possible generalization capability [6, 10]. The main disadvantage prior to training the network is that training samples must be collected for 
each time step ' $t$ ' to define quantitative error signals. This sampling process is reduced by using reinforcement learning for qualitative success or failure feedback at the end of the control sequence.

\section{Experimental Results}

The experimental procedure executed for both software agent platforms are as elaborated below:

1. The determination of a successful pseudo-random curve shown on this page (A) and generalized trajectory curve on the current page (B) of an actuator passing through a SANET environment in $20 \mathrm{~cm} \times 20 \mathrm{~cm}$ space.

2. The maximum processor load was calculated using VisualVM Profiler Agent, which calculates processor load of the program, with 30 experimental repetitions for each node density to determine average processor loads.

3. The software platform is a Windows 7 Enterprise operating system, running Java Standard Edition 1.6 (r25); the hardware specifications is a Pentium Dual-core Processor (2.1Ghz) with $4 \mathrm{~GB}$ of memory.

4. The number of total agents consist of the sensor network population in the SANET environment, with density and spread determined in a pseudorandom fashion via the Mersenne-twister method.

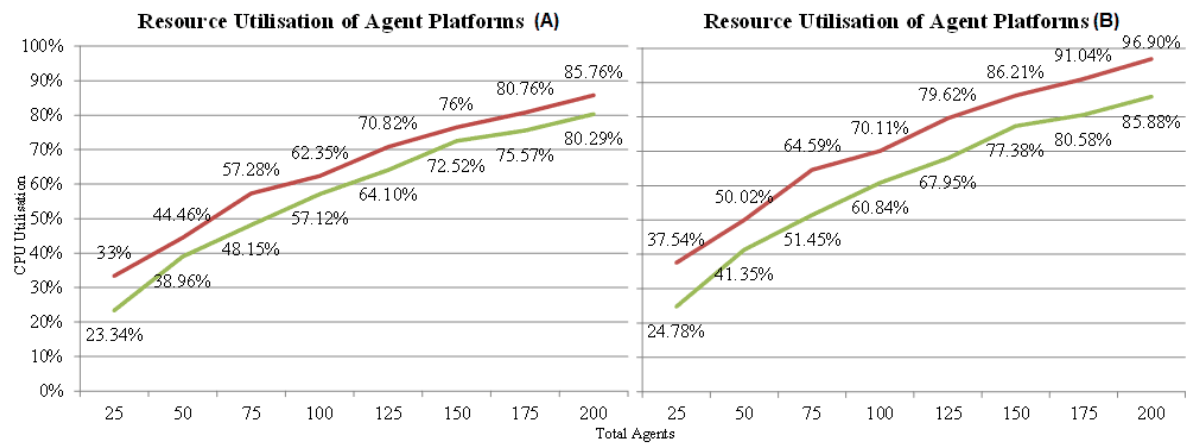

Legend: Red - AnyLogic Framework; Green - Jadex BDI Framework

Fig. 3. (a) Resource Utilization for Pseudo-random Trajectories; (b) Resource Utilization for Linear Trajectories

As evident from the results, processor load increases as sensor node densities increase, because the number of obstacles reduces the opportunity for a direct actuator trajectory. In addition, linear trajectories will require less calculation processing than pseudo-random trajectories due to simpler path calculation processes. It is noted that linear trajectory results are included as a control sample for comparative study, but in reality such actuator navigation paths is unrealistic for actual training scenarios. The interesting result is that for pseudo-random trajectories, AnyLogic requires on average 30\% processing cycles than Jadex for 
pseudo-random paths, and 25\% more processing for linear paths. As summarized on this page, the justification for these results is due to the flexibility afforded in the design of the two agent-based frameworks: while AnyLogic encapsulates code generation within its codified framework, provisioning Jadex for additional service activation is simplified due to its open-porting facilities, thus enabling distributed load handling within the Jadex engine. Thus, the BDI architecture of Jadex allows for direct relational mapping with the SANET environment, making system design flexible for service extensions in future.

Table 1. Evaluation Summary of Agent-based Framework for SANETs

\begin{tabular}{|c|c|c|}
\hline & AnyLogic Framework & Jadex BDI Framework \\
\hline $\begin{array}{c}\text { Software } \\
\text { Evaluation }\end{array}$ & $\begin{array}{l}\text { - } \text { Closed Source Java-based } \\
\text { Framework needs BDI } \\
\text { compliant integration design } \\
\text { - Eclipse user interface with } \\
\text { encapsulated code-generation } \\
\text { - No native support for general } \\
\text { purpose computing on GPU's } \\
\text { (OpenCL, CUDA) }\end{array}$ & $\begin{array}{l}\text { - Open Source Java-based } \\
\text { Framework is BDI compliant } \\
\text { based on Jade Engine } \\
\text { - Open-port libraries enable } \\
\text { customization and adaption to } \\
\text { heuristic tracking functions } \\
\text { - Potential for supporting general } \\
\text { purpose computing for GPU's }\end{array}$ \\
\hline $\begin{array}{l}\text { Technology } \\
\text { Assessment }\end{array}$ & $\begin{array}{l}\text { - Closed framework and limited } \\
\text { customization for parallel tasks } \\
\text { makes heuristic integration } \\
\text { difficult for post-processing }\end{array}$ & $\begin{array}{l}\text { - Flexible framework allows for } \\
\text { customization of distributed } \\
\text { processing tasks for a specific } \\
\text { domain and environment space }\end{array}$ \\
\hline
\end{tabular}

\subsection{Future Work}

Future experimental work to consider in the software agent test-bed include:

- Utilizing OpenCL and CUDA processing libraries to improve parallel processing performance of tasks and heuristics;

- Improving experimental models by developing realistic a 3D mesh model to visualize real-life organic concerns to augment experimental environment;

- Optimization approach tailored for a particular service, in terms of suitability for a particular domain context (i.e. random, ad-hoc environments); and

- Development of agent-based middleware in constrained platforms, such as embedded Java frameworks (Java ME) to assess performance characteristics in ARM RISC-based hardware architectures.

\section{Conclusion}

The suitability of an agent-based framework should allow for distributed processing tasks from a multi-processor perspective, not just in terms of multi-threaded 
capability. Embedded hardware capability is becoming multi-processor intensive, and software agent-based architectures need to cater for this development shift. The main benefit of software agents utilizing multi-processor architectures is to enhance real-time performance while reducing processing load per processor, thus reducing overall power consumption. Furthermore, an agent-based framework should have open application interfaces for augmenting middleware-class services, as emerging software technologies can be integrated in a seamless and coordinated manner. The quality attributes that drive efficient software agent architectures will ultimately impact on the system environment such that within the near future, laparoscopic surgical training systems will have scope to embed software agents within the physical domain.

\section{References}

[1] Feng, C., Rozenblit, J.W., Hamilton, A.J.: A computerized assessment to compare the impact of standard. stereoscopic and high definition laparoscopic monitor displays on surgical technique, Surgical Endoscopy 24(11), 2743-2748 (2010), doi:10.1007/s00464-010-1038-6

[2] Feng, C., Rozenblit, J.W., Hamilton, A.J.: A Hybrid View in a Laparoscopic Surgery Train- ing System. In: Proc. of the 14th IEEE Intl. Conference and Workshops on the Engineering of Computer Based Systems (ECBS 2007), Tucson, Arizona, pp. 339-348 (2007)

[3] Feng, C., Rozenblit, J.W., Hamilton, A.J., Wytyczak-Partyka, A.: De_ning Spatial Regions in Computer-assisted Laparoscopic Surgical Training. In: Proc. of the 16th IEEE Intl. Conf. on Engineering of Computer Based Systems (ECBS 2009), San Francisco, USA, pp. 176-183 (2009)

[4] Halkidi, M., Batistakis, Y., Vazirgiannis, M.: Clustering Validation Techniques. Intelligent Information Systems Journal 17(2-3), 107-145 (2001)

[5] Kohonen, T.: Self-Organizing Maps, 3rd edn. Springer, New York (2000)

[6] Low, K.H., et al.: An Ensemble of Cooperative Extended Kohonen Maps for Complex Robot Motion Tasks. Neural Computation 17(6), 1411-1445 (2005)

[7] Low, K.H., et al.: Task Allocation via Self-organizing Swarm Coalitions in Distributed Sensor Networks. In: 19th Artificial Intelligence Conference, pp. 28-33 (2004)

[8] Rao, M., Georgeff, P.: BDI-agents: From Theory to Practice. In: Proceedings of the 1st International Conference on Multiagent Systems (ICMAS 1995), San Francisco, USA (1995)

[9] Ritter, H., Schulten, K., Denker, J.S.: Topology Conserving Mappings for Learning Mo- tor Tasks: Neural Networks for Computing. In: Proceedings of 151st Conference, pp. 376-380. American Institute of Physics, Snowbird (1986)

[10] Sharkey, A., et al.: Combining Diverse Neural Nets. Knowledge Eng. Rev., 231-247 (1997)

[11] Wytyczak-Partyka, A., Nikodem, J., Klempous, R., Rozenblit, J., Klempous, R., Rudas, I.: Safety Oriented Laparoscopic Surgery Training System. In: MorenoDíaz, R., Pichler, F., Quesada-Arencibia, A. (eds.) EUROCAST 2009. LNCS, vol. 5717, pp. 889-896. Springer, Heidelberg (2009)

[12] Yang, L., Feng, C., Peng, J., Rozenblit, J.W.: A Multi-modality Framework for Energy Efficient Tracking in Large Scale Wireless Sensor Networks. In: Proc. of the 2nd IEEE Intl. Conference on Networking, Sensing and Control, Ft. Lauderdale, FL, USA, pp. 916-921 (2006) 